\title{
MAPEAMENTO DA CULTURA CAFEEIRA POR MEIO DE CLASSIFICAÇÃO AUTOMÁTICA UTILIZANDO ATRIBUTOS ESPECTRAIS, TEXTURAIS E FATOR DE ILUMINAÇÃO
}

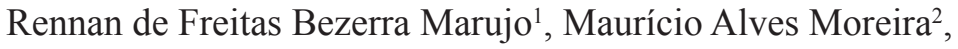 \\ Margarete Marin Lordelo Volpato ${ }^{3}$, Helena Maria Ramos Alves ${ }^{4}$
}

(Recebido: 07 de março de 2016; aceito: 12 de setembro de 2016)

\begin{abstract}
RESUMO: O café, importante produto nas exportações brasileiras, necessita de constante monitoramento para que os sistemas de previsão de safras existentes sejam confiáveis. Imagens orbitais de média resolução espacial são ferramentas com grande potencial para mapeamento do uso do solo e identificação de culturas agrícolas. Nesta pesquisa, visando o mapeamento de áreas cafeeiras, avaliou-se o desempenho da classificação baseada em objetos, associada a técnicas de mineração de dados, aplicada em imagens OLI/Landsat-8. Foram feitas três classificações automáticas, a primeira constando exclusivamente atributos espectrais, a segunda acrescentando atributos texturais e a terceira, incluindo também classes de iluminação do terreno. Foram utilizadas seis imagens multiespectrais, datadas de três diferentes estádios fenológicos da cultura: frutificação, granação e repouso. A validação das classificações foi feita por meio do Método de Monte Carlo utilizando como referência mapas visualmente interpretados. As classificações feitas exclusivamente com atributos espectrais resultaram, para a classe café, exatidão média de $57 \%$. Não houve estádio fenológico que proporcionasse maior exatidão à classe café, entretanto ao incluir os atributos texturais, a exatidão da classe café melhorou para $76 \%$. Assim, observa-se que atributos texturais mostraram-se importantes para detecção automática de áreas cafeeiras.
\end{abstract}

Termos para indexação: Sensoriamento remoto, SRTM, Landsat-8.

\section{COFFEE CROP DETECTION BY AUTOMATIC CLASSIFICATION USING SPECTRAL AND TEXTURAL ATTRIBUTES AND ILLUMINATION FACTOR}

\begin{abstract}
Coffee, an important product in Brazilian exports, needs constant monitoring, so that systems and forecasting of existing crops can be reliable. Orbital imagery of medium spatial resolution are tools with great potential for land use mapping and identification of agricultural crops. This research evaluated the performance of the object based classification, applied in OLI/Landsat-8 images, with the purpose of mapping of coffee crops. Three analyzes were made, the first one using exclusively spectral attribute, the second one including textural attributes and the third also considering illumination classes. Six OLII Landsat-8 multispectral images were used, representing three different coffee phenological stages: fructification, graining and rest. The validation of the classifications was performed by the Monte Carlo method using reference images obtained by visual interpretation. The classification using exclusively spectral attributes resulted an accuracy of $57 \%$ for coffee class. There was no phenological stage that provided greater accuracy to the coffee class in the automatic classification of OLI/Landsat-8 images. The results demonstrate that texture is important for coffee detection, thus visual interpretation remains an important step to minimize classification errors.
\end{abstract}

Index-terms: Remote sensing, SRTM, Landsat-8.

\section{INTRODUÇÃO}

O café é uma das bebidas mais consumidas no mundo (LUDWIG et al., 2014), com uma média de produção de aproximadamente 5,9 milhões de toneladas por ano (BATTISTA; FINO; MANCINI, 2016) e o Brasil responde por mais de $30 \%$ dessa produção (INTERNATIONAL COFFEE ORGANIZATION - ICO, 2016). Aproximadamente $50 \%$ da produção do café está concentrada no estado de Minas Gerais (COMPANHIA NACIONAL DE ABASTECIMENTO - CONAB, 2015), havendo entre os maiores produtores municípios mais planos como, Três Pontas e outros com relevo montanhoso, por exemplo Machado, ambos no sul do estado. Apesar de sua importância econômica, os métodos utilizados para o acompanhamento da área plantada, da produtividade e produção, diferem entre instituições governamentais e de iniciativa privada (SOUZA et al., 2012). Portanto, o monitoramento do agroecossistema cafeeiro, bem como o acompanhamento das safras de café no Brasil, carece de metodologias concisas e padronizadas.

Conhecer a distribuição espacial das lavouras cafeeiras é vital para prever e planejar

\footnotetext{
${ }^{1,2}$ Instituto Nacional de Pesquisas Espaciais/INPE - Departamento de Sensoriamento Remoto/DSR - Cx. P. 515 - 12.201-970 São José dos Campos - SP - rennan.marujo@inpe.br, mauricio@dsr.inpe.br

${ }^{3}$ Empresa de Pesquisa Agropecuária de Minas Gerais/EPAMIG - Laboratório de Geoprocessamento/ GEOSOLOS - Cx. P. 176 37.200-000 - Lavras - MG - margarete@epamig.ufla.br

${ }^{4}$ Empresa Brasileira de Pesquisa Agropecuária/EMBRAPA CAFÉ - Laboratório de Geoprocessamento/ GEOSOLOS - Cx. P. 176

37.200-000 - Lavras - MG - helena.alves@embrapa.br
} 
ações em escala municipal, estadual e federal (MOREIRA; BARROS; RUDORFF, 2008). Imagens de satélite com média resolução espacial e técnicas de geoprocessamento são ferramentas com grande potencial para padronizar metodologias confiáveis que possibilitem o monitoramento do agroecossistema, e o acompanhamento das safras do café (MACHADO et al., 2010; MOREIRA et al., 2007; MOURA et al., 2009). Dentre as imagens mais utilizadas para mapeamento de culturas agrícolas (ALI; ALIREZA; POURMANAFI, 2016; PEÑA; BRENNING, 2015; XIE et al., 2008), pode-se citar as do programa Landsat, que produz o mais longo registro de imagens orbitais (BANSKOTA et al., 2014).

Dentre as principais dificuldades no mapeamento automático de áreas cafeeiras por meio de imagens orbitais, exaltam-se a semelhança no comportamento espectral do café com outras fisionomias (CORDERO-SANCHO; SADER, 2007; VIEIRA et al., 2006), a variação em função de fatores da planta (sanidade, estado nutritivo e variedade), dos tratos culturais e do manejo da cultura (ADAMI et al., 2007) e do sombreamento proporcionado por seu cultivo em áreas montanhosas (VIEIRA et al., 2006). Além desses fatores há também o ciclo fenológico da planta, que varia no decorrer de dois anos no caso do café (CAMARGO, 1985) e que é pouco explorado no contexto da identificação automática de áreas cafeeiras.

As dificuldades em classificar automaticamente áreas cafeeiras proporcionam baixa exatidão de mapeamento, com muitos erros de inclusão e omissão (ANDRADE et al., 2012; CORDERO-SANCHO; SADER, 2007; GIROLAMO NETO et al., 2015). Deste modo, em áreas planas e homogêneas o mapeamento de áreas cafeeiras pode ser realizado exclusivamente com dados orbitais (LELONG; CHANE, 2003), entretanto para áreas montanhosas, ou com mistura de culturas, os mapeamentos precisam ser feitos por meio de abordagem visual (GUHL, 2004; MOREIRA; BARROS; RUDORFF, 2008). Há também as abordagens híbridas, que utilizam dados orbitais e verificação em campo (VERDUZCO; PIZAÑA; BELLO, 2012).

Alguns trabalhos demonstram que o uso de informação topográfica na identificação automática de áreas cafeeiras, pode amenizar os efeitos do sombreamento, causado por revelo acentuado (CORDERO-SANCHO; SADER, 2007; LAMPARELLI; NERY; ROCHA, 2011; VIEIRA et al., 2006). Outros sugerem a utilização de atributos texturais para melhor identificar as áreas cafeeiras (ANDRADE et al., 2012; GIROLAMO NETO et al., 2015; LELONG; CHANE, 2003). A análise baseada em objetos (da sigla em inglês, GEOBIA: Geographic objectbased image analysis) tem ganhado ênfase por possibilitar o uso de atributos relacionais, de forma e de textura (ADDINK; COILLIE; DE JONG, 2012), mostrando-se promissora na identificação de outras culturas (LUZ; ANTUNES; TAVARES JÚNIOR, 2010), como cana de açúcar (VIEIRA et al., 2012; ZHOU et al., 2015) e soja (LI et al., 2015; SCHULTZ et al., 2014; SILVA JUNIOR; FRANK; RODRIGUES, 2014), sendo pouco explorada no contexto do café (MARUJO, 2013; SANTOS et al., 2012; SARMIENTO et al., 2014).

Devido aos desafios existentes no mapeamento automático de áreas cafeeiras, objetivou-se classificar automaticamente áreas cafeeiras no município de Machado, MG, utilizando o método de classificação baseada em objetos associada a técnicas de mineração de dados, aplicada em imagens OLI/Landsat-8 e avaliar a influência dos atributos texturais e do fator de iluminação na exatidão do mapeamento.

\section{MATERIAL E MÉTODOS}

O desenvolvimento deste trabalho teve como área de estudo o município de Machado, um dos vinte maiores produtores de café do estado de Minas Gerais (INSTITUTO BRASILEIRO DE GEOGRAFIA E ESTATÍSTICA - IBGE, 2012). Trata-se de uma região montanhosa que compreende uma área territorial de $520 \mathrm{~km}^{2}$ (IBGE, 1990) delimitada pelas coordenadas $21^{\circ} 42^{\prime} 05^{\prime \prime} \mathrm{S}$ e $21^{\circ} 31^{\prime} 10^{\prime \prime} \mathrm{S}$ e entre $46^{\circ} 02^{\prime} 08^{\prime \prime}$ WGr e 45\%47'30"WGr. Machado encontra-se na região sul do estado e é caracterizado por relevo montanhoso, com altitudes de 780 a 1260 metros (ANDRADE et al., 2012).

\subsection{Metodologia}

A metodologia adotada nesta pesquisa apresenta quatro etapas conforme ilustrado na Figura 1.

Foram utilizadas seis imagens OLI/ Landsat-8 referentes às produções cafeeiras de 2013/2014 e 2014/2015, de modo a representar um ciclo fenológico inteiro da cultura. Durante a seleção das imagens buscou-se condições climáticas ideais para o mapeamento, ou seja, imagens sem nuvens. $\mathrm{O}$ estudo compreendeu um intervalo de dois anos, de modo que se tentou estabelecer um intervalo equidistante de quatro meses entre cada imagem. 


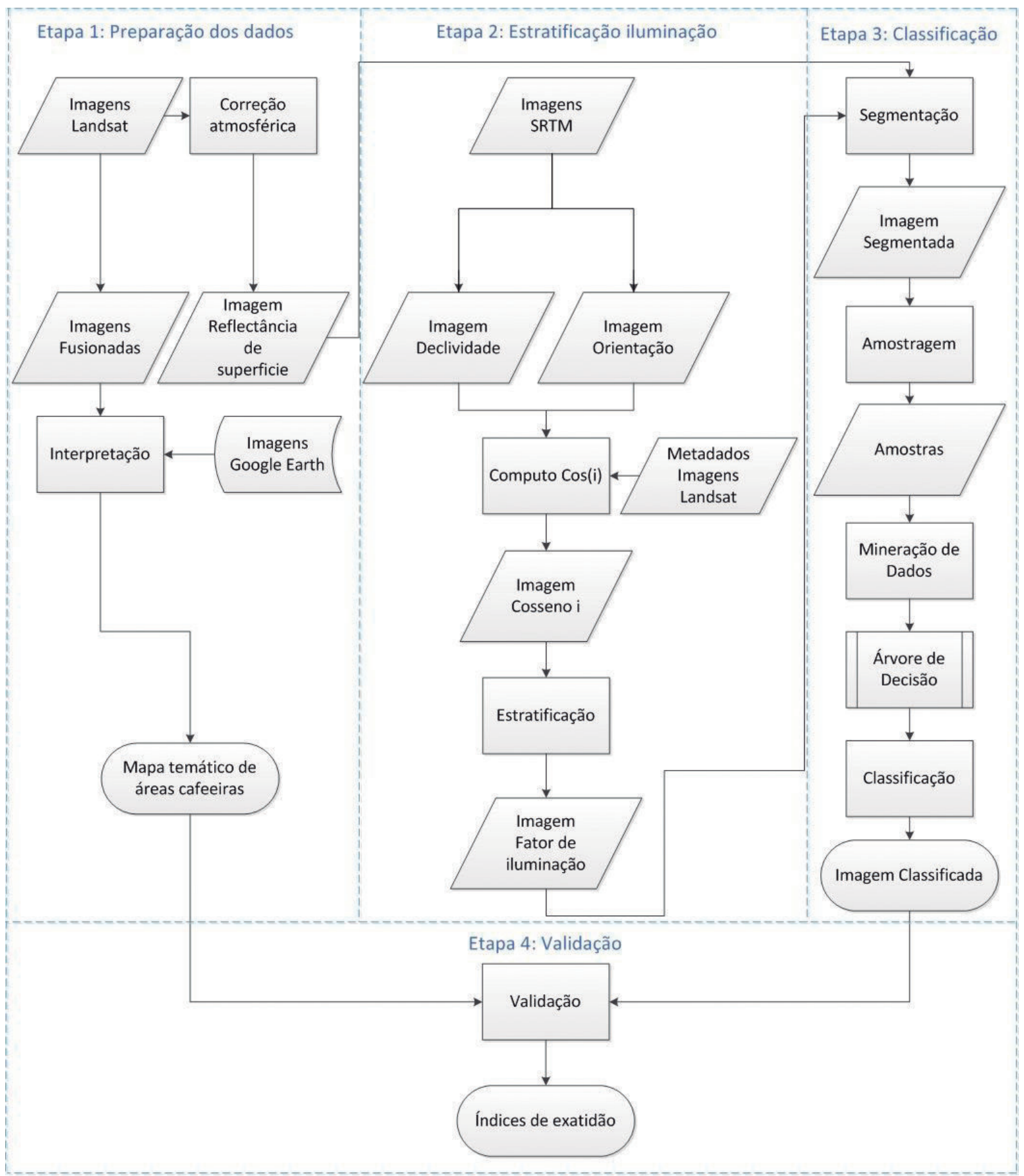

FIGURA 1 - Fluxograma de atividades desenvolvidas no trabalho, composta por quatro etapas: 1-Preparação dos dados; 2-Estratificação das classes de iluminação; 3-Classificação baseada em objetos; e 4-Validação.

Tendo conhecimento de que a planta sofre variações ao decorrer do ano, devido às alterações de estádio fenológico, neste estudo representaramse os estádios fenológicos da frutificação, maturação e repouso (CAMARGO, 1985). Também foram utilizados dados de altimetria da
Missão Topográfica Radar Shuttle (SRTM) para informações sobre o relevo. Tanto as imagens OLI/ Landsat-8 quanto às imagens SRTM, apresentam 30 metros de resolução espacial e foram obtidas gratuitamente no site Earth Explorer (www. earthexplorer.usgs.gov). 


\subsection{Etapa 1: Preparação dos dados}

As imagens utilizadas no estudo foram obtidas georreferenciadas. Para que os dados multitemporais fossem comparáveis, fez-se o procedimento de correção dos efeitos atmosféricos para redução de ruídos nas imagens nas imagens (SONG et al., 2001). A correção atmosférica foi realizada pelo método Fast Line-of-sight Atmospheric Analysis of Hypercubes (Flaash), que é baseada no modelo de transmissão atmosférica Moderate Resolution Atmospheric Radiance and Transmittance (MODTRAN), utilizando dados atmosféricos observados no momento da aquisição da imagem para inferir a reflectância de superfície (CALLIECO; DELL'ACQUA, 2011).

Para interpretação visual das imagens, foi feita a fusão pelo método Gram-Schmidt, no qual gera-se uma banda pancromática artificial utilizando as bandas multiespectrais, de modo que a pancromática artificial é substituída por uma imagem pancromática verdadeira, resultando assim em uma imagem com melhor qualidade visual (PESCK; DISPERATI; SANTOS, 2011). A interpretação visual foi feita conforme recomendações de Moreira, Barros e Rudorff (2008), analisado os talhões de café nas imagens OLI/Landsat-8 (20/11/2013 e 21/07/2015) e conferindo, nas imagens de alta resolução espacial disponíveis no Google Earth. Ao se identificar na imagem de alta resolução espacial uma lavoura cafeeira, desenhava-se um polígono nos contornos do alvo e este era associado à classe café. Embora as imagens do Google Earth fossem obtidas em outra época (04/08/14), essa comparação pôde ser feita porque o café é uma cultura perene (ROSA, 2007).

\subsection{Etapa 2: Estratificação de relevo em Classes de Iluminação}

Um dos fatores que podem influenciar negativamente na identificação automática de áreas cafeeiras em imagens orbitais é o sombreamento causado por revelo acentuado (CORDEROSANCHO; SADER, 2007; MOREIRA; BARROS; RUDORFF, 2008; VIEIRA et al., 2006). Uma das opções para minimizar a influência do sombreamento nas imagens orbitais é a estratificação em classes de iluminação (LAMPARELLI; NERY; ROCHA, 2011; MEYER et al., 1993; MOREIRA; VALERIANO, 2014).

A partir dos dados de elevação SRTM, calculou-se declividade e a orientação de vertentes (por vezes também chamada orientação da face do relevo). Aliando estes dados aos metadados das imagens OLI/Landsat-8 (ângulo solar azimutal e ângulo solar zenital), contidos na Tabela 1, calculou-se o fator de iluminação $\cos (i)$ para cada imagem por meio da Equação 1.

$\cos (\theta o)=\cos (\theta s) \cos (\theta t)+\cos (\theta s) \cos (\varphi s-\varphi t)$,

em que, $\cos (\theta o)$ é o fator cosseno (fator de iluminação), $\theta s$ é o ângulo solar zenital, $\theta t$ é o ângulo zenital da normal à superfície do terreno (que corresponde à declividade), $\varphi s$ é o ângulo solar azimutal e $\varphi t$ é o ângulo azimutal da normal à superfície (que corresponde à orientação de vertente) (VALERIANO, 2011).

Meyer et al. (1993) estratificam o relevo em dez classes de iluminação com variação equidistante de 0,1 . Neste estudo, visando uma metodologia operacional, optou-se por estratificar o fator de iluminação em cinco classes de iluminação, adotando intervalos equidistantes de 0,2 , conforme dispostos na Tabela 2 e em duas classes de iluminação, de 0,0-0,5 e 0,5-1, respectivamente.

\subsection{Etapa 3: Classificação baseada em objetos}

Para realizar a classificação baseada em objetos, primeiramente foi necessário definir os objetos durante o processo de segmentação. Uma A avaliação da segmentação é feita por interprete humano (ANTUNES, 2003; GAMANYA; DE MAEYER; DAPPER, 2007; PRATT, 2007), visando obter segmentos adequados às feições dos talhões cafeeiros e de modo que um segmento não aglomere diferentes alvos em um mesmo objeto. Empiricamente estabeleceu-se para o algoritmo multirresolução (BAATZ; SCHAPE, 2000) os parâmetros: fator de similaridade 30 , forma 0,6 e compacidade 0,5 nas bandas: NDVI, pancromática, vermelho, e infravermelho próximo (bandas em que a vegetação apresentou maior caracterização).

Após a segmentação, tornou-se possível a extração dos atributos dos segmentos. Foram realizadas três extrações com diferentes atributos, conforme Tabela 3. Para a primeira classificação foram extraídos os atributos: média espectral de cada banda, desvio padrão médio em cada banda, brilho, diferença máxima, compacidade, retangularidade, elipsidade, assimetria e densidade. Para a segunda classificação além dos atributos citados foram extraídos atributos de textura (HARALICK; SHANMUGAN; DINSTEIN, 1973): segundo momento angular, contraste, correlação, dissimilaridade, entropia, homogeneidade, média e desvio padrão. 
TABELA 1 - Imagens OLI/Landsat-8 (219|075) utilizadas e metadados.

\begin{tabular}{|c|c|c|c|c|c|}
\hline Imagem & $\begin{array}{c}\text { Data } \\
(\mathrm{dd} / \mathbf{m m} / \mathbf{a a})\end{array}$ & $\begin{array}{c}\text { Estádio } \\
\text { fenológico }\end{array}$ & $\begin{array}{c}\text { Horário de } \\
\text { aquisição } \\
\text { (hh:mm:ss) }\end{array}$ & $\begin{array}{c}\text { Ângulo } \\
\text { solar Azimutal } \\
\left({ }^{\circ}\right)\end{array}$ & $\begin{array}{c}\text { Ângulo } \\
\text { Solar } \\
\text { Zenital ( }\left(^{\circ}\right)\end{array}$ \\
\hline 1 & $20 / 11 / 2013$ & Frutificação & $13: 05: 34$ & 90,68 & 65,47 \\
\hline 2 & $08 / 02 / 2014$ & Granação & $13: 04: 54$ & 83,93 & 57,59 \\
\hline 3 & 02/07/2014 & Repouso & $13: 03: 44$ & 36,18 & 36,04 \\
\hline 4 & $07 / 11 / 2014$ & Frutificação & 13:04:11 & 82,58 & 64,86 \\
\hline 5 & $10 / 01 / 2015$ & Granação & $13: 04: 00$ & 96,83 & 60,32 \\
\hline 6 & $21 / 07 / 2015$ & Repouso & $13: 03: 32$ & 38,66 & 37,59 \\
\hline
\end{tabular}

TABELA 2 - Estratificação das imagens fator de iluminação em cinco classes.

\begin{tabular}{ccc}
\hline Classe de iluminação & Intervalo do fator de iluminação & Nome da classe \\
\hline 1 & $0,0-0,2$ & muito sombreado \\
2 & $0,2-0,4$ & sombreado \\
3 & $0,4-0,6$ & Pouco iluminado \\
4 & $0,6-0,8$ & iluminado \\
5 & $0,8-1,0$ & Muito iluminado \\
\hline
\end{tabular}

Por fim, para a terceira classificação, foram extraídos os mesmos atributos da segunda classificação, acrescentando-se as classes de iluminação.

Para treinamento do classificador foram amostrados aleatoriamente, a partir do mapa de referência, 10.000 segmentos, referentes às classes café e não café. O treinamento fez-se por meio do algoritmo C4.5 de Quinlan (1993) em sua implementação no software WEKA (HALL et al., 2009). O algoritmo C4.5, verifica em um conjunto de dados os atributos mais importantes para determinação de uma classe, utilizando para isso o conceito de entropia (QUINLAN, 1993). Como resultado da exploração dos dados o algoritmo C4.5 gera uma árvore de decisão (DELGADO et al., 2012). A árvore de decisão, contendo as regras de classificação, pode então ser aplicada a alvos não classificados resultando assim em um alvo classificado (KIM, 2016). Esse procedimento de treinamento e classificação foi replicado para as demais datas.

\subsection{Etapa 4: Validação}

Para validar as classificações empregouse o Método de Monte Carlo (MMC) que é um método estatístico de padrão estocástico que obtém parâmetros de uma função por meio da repetição de um cálculo diversas vezes (LANDAU; BINDER, 2009). Utilizando os mapas de interpretação visual, após 100 sorteios de 1.000 pontos calculou-se uma matriz de confusão média para cada classificação. Para avaliar a exatidão das classificações, as matrizes foram utilizadas para calcular o Índice Global (IG) e a exatidão para a classe café. $O$ índice global, também chamado de exatidão avalia a porcentagem de acerto de um mapa e é dado pela equação 2 e a exatidão de uma classe específica, invés de avaliar a exatidão do mapa inteiro, avalia a porcentagem de acerto de uma única classe.

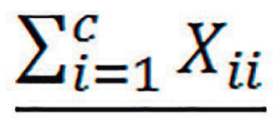

\section{$n$}

em que $c$ representa a última classe; $n$ representa o total de classes e $i$ o índice na matriz de confusão. 
TABELA 3 - Tabela de atributos extraídos dos segmentos da imagem, para posteriores classificações.

\begin{tabular}{lll}
\hline \multicolumn{1}{c}{ Atributos Classificação 1 } & \multicolumn{1}{c}{ Atributos Classificação 2 } & \multicolumn{1}{c}{ Atributos Classificação 3 } \\
\hline - Média espectral de cada banda $(*)$ & - Atributos Classificação 1 & - Atributos Classificação 1 \\
- Desvio padrão médio em cada banda $(*)$ & - Textura Segundo momento angular & - Atributos Classificação 2 \\
Brilho & - Textura Contraste $(* *)$ & - Classes de iluminação \\
- Diferença máxima & - Textura Correlação $(* *)$ & \\
Compacidade & - Textura Dissimilaridade $(* *)$ & \\
- Retangularidade & - Textura Entropia $(* *)$ & \\
- Elipsidade & - Textura Homogeneidade $(* *)$ \\
- Assimetria & - Textura Média $(* *)$ \\
- Densidade & - Textura Desvio Padrão $(* *)$ \\
\hline
\end{tabular}

* Para bandas do azul, verde, vermelho, infravermelho próximo, swir1, swir2 e ndvi

** Para bandas do azul, verde, vermelho, infravermelho próximo, swir1, swir2 e ndvi e pan

\section{RESULTADOS E DISCUSSÃO}

\subsection{Interpretação visual}

Como resultado da interpretação visual, obteve-se para cada data, um mapa temático de referência das áreas cafeeiras. A imagem temática de referência da primeira data $(20 / 11 / 2013)$ pode ser observada na Figura 2.

Por análise das imagens, foi possível verificar que aproximadamente $22,2 \%$ da área do município de Machado consistem de áreas cafeeiras e estas estão distribuídas por todo o município, havendo maior concentração na porção centro-oeste. Segundo IBGE (2012) o percentual de área de produção cafeeira em relação à área do município no ano de 2012 era de 17\%. Moreira, Bernardes e Saito (2013) obtiveram para o mesmo ano $20,5 \%$ de área com café. Esses resultados demonstram a grande variação das informações nos dados entre diferentes instituições citados por Souza et al. (2012). Considerando o relevo acidentado do munícipio é possível que o processo de interpretação visual tenha superestimado a área cafeeira do município, entretanto não há um mapeamento que possa ser tomado por verdade absoluta. Deste modo, considerando que não houve mudança na área plantada de café entre o ano de 2012 e 2013, o mapeamento forneceu diferença de 5\% (2915 ha) para com os dados do IBGE (2012) e 1,7\% (992 ha) para com os dados de Moreira, Bernardes e Saito (2013).

\subsection{Estratificação em classes de iluminação}

$\mathrm{Na}$ Figura $3 \mathrm{a}$ pode-se observar parte de uma imagem fator de iluminação, e na Figura 3b essa mesma imagem estratificada em classes de iluminação.

Na Tabela 4 são apresentados os resultados obtidos a partir do cruzamento das imagens de fator de iluminação estratificadas em classes de iluminação com as áreas de café obtidas no mapa de referência. Nota-se que na primeira e quarta imagens, referentes ao primeiro período de frutificação, praticamente toda a área de estudo encontra-se na classe muito iluminado ou iluminado. Segundo Camargo (1985), nesse período é quando mais ocorrem atividades fotossintetizantes, justamente devido à maior iluminação diária. No período da granação, a atividade fotossintética diminui, mas as áreas cafeeiras ainda se encontram em áreas com privilégio de iluminação. Apenas no período do repouso, algumas áreas cafeeiras estavam em situação bastante sombreada, entretanto nesta etapa do ciclo fenológico do café a atividade fotossintética é reduzida, pois está ocorrendo o processo de auto-poda (CAMARGO; CAMARGO, 2001).

A alteração mais perceptível de classes de iluminação em um mesmo estádio fenológico ocorreu no estádio da granação, onde o café passou de $31 \%$ na classe iluminado para $21 \%$ no intervalo de um ano e um mês, havendo a maior parte deste café sido incluída na faixa com fator de iluminação entre 0,8 e 1,0 (classe muito iluminado). Tal diferença pode ser explicada pelo ângulo solar azimutal, que teve variação significante (aproximadamente $13^{\circ}$ ). 


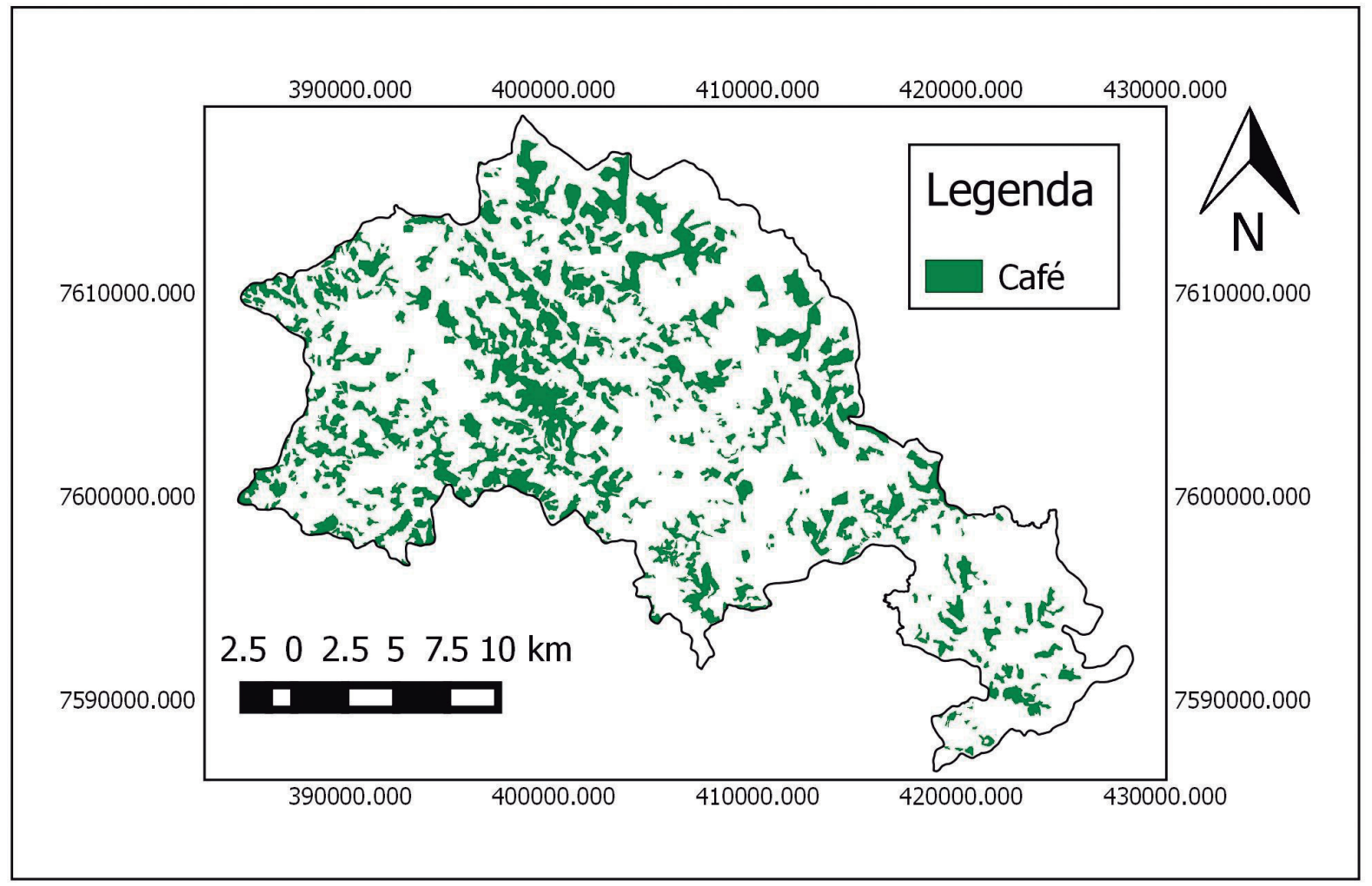

FIGURA 2 - Mapa temático resultante da interpretação visual de imagem OLI/Lansat-8 para o Município de Machado em 20/11/2013.
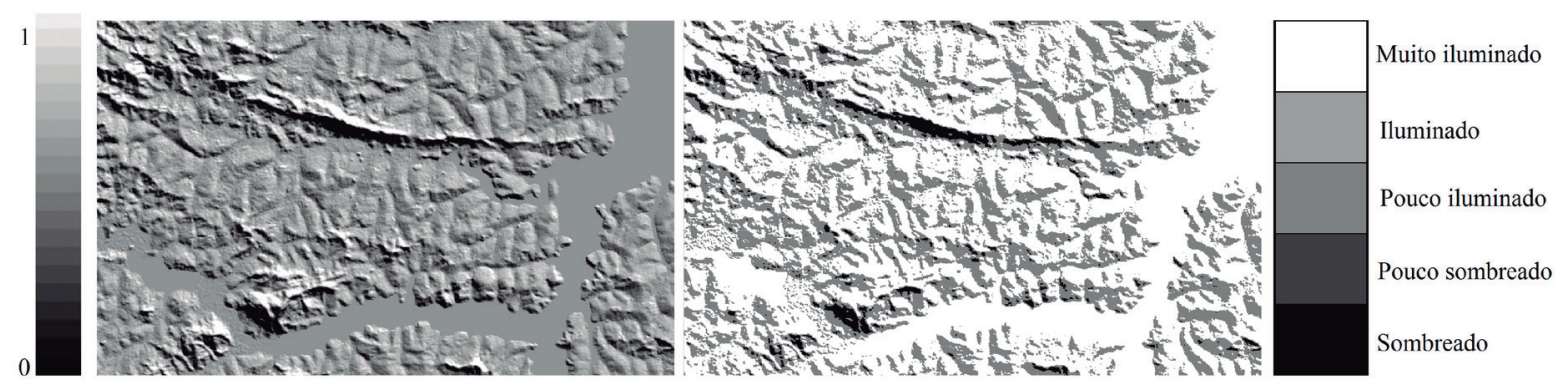

FIGURA 3 - Fator de iluminação (a) e estratificação em classes de iluminação (b).

Isso foi devido à diferença na data de aquisição dasimagens, que foi de aproximadamente um ano e um mês. Essa diferença na data de aquisição não foi possível de ser amenizada, pois as datas mais próximas continham nuvens, o que dificultava a análise da superfície terrestre. Já no período de repouso, a diferença de classes de iluminação entre os anos analisados foi sútil, havendo incremento das áreas cafeeiras em locais sombreados.

\subsection{Validação}

Uma vez que aproximadamente $22,2 \%$ da área estudada eram talhões cafeeiros e 77,8\% não, o índice global apresentar valores altos não indica necessariamente uma boa classificação, porque as áreas de não café podem mascarar os erros cometidos na classe café. Assim, o acerto da classe café deve ser analisado por meio da exatidão da classe café. 
TABELA 4 - Distribuição dos cafezais do município de Machado (MG) em classes de iluminação no período entre 20/11/2013 e 21/07/2015.

\begin{tabular}{|c|c|c|c|c|c|c|}
\hline \multicolumn{7}{|c|}{ Percentagem de área de café sob sombreamento no município de Machado (MG) } \\
\hline & \multicolumn{6}{|c|}{ Estádio Fenológico } \\
\hline & Frutificação & Granação & & & epouso & \\
\hline Data & $20 / 11 / 2013$ & $07 / 11 / 2014$ & $08 / 02 / 2014$ & $10 / 01 / 2015$ & $02 / 07 / 2014$ & $21 / 07 / 2015$ \\
\hline Azimute & $90,68^{\circ}$ & $83,93^{\circ}$ & $36,18^{\circ}$ & $82,58^{\circ}$ & $96,83^{\circ}$ & $38,66^{\circ}$ \\
\hline Elevação solar & $65,47^{\circ}$ & $57,59^{\circ}$ & $36,04^{\circ}$ & $64,86^{\circ}$ & $60,32^{\circ}$ & $37,59^{\circ}$ \\
\hline $\begin{array}{l}\text { muito sombreado } \\
\qquad(0,0-0,2)\end{array}$ & $0,0 \%$ & $0,0 \%$ & $0,0 \%$ & $0,0 \%$ & $0,0 \%$ & $0,0 \%$ \\
\hline $\begin{array}{l}\text { sombrado } \\
(0,2-0,4)\end{array}$ & $0,0 \%$ & $6 \%$ & $0,0 \%$ & $0,0 \%$ & $8,0 \%$ & $6,0 \%$ \\
\hline $\begin{array}{l}\text { pouco iluminado } \\
(0,4-0,6)\end{array}$ & $0,0 \%$ & $36 \%$ & $1,0 \%$ & $0,0 \%$ & $41 \%$ & $36 \%$ \\
\hline $\begin{array}{l}\text { iluminado } \\
(0,6-0,8)\end{array}$ & $8,0 \%$ & $53 \%$ & $31 \%$ & $21 \%$ & $47 \%$ & $53 \%$ \\
\hline $\begin{array}{l}\text { muito iluminado } \\
\quad(0,8-1)\end{array}$ & $92 \%$ & $5,0 \%$ & $68 \%$ & $79 \%$ & $4,0 \%$ & $5,0 \%$ \\
\hline
\end{tabular}

Na Tabela 5 podem-se observar os índices globais e as exatidões para a classe café, ambos obtidos por meio do Método de Monte Carlo (MMC). Nota-se que quando são utilizados apenas atributos espectrais para classificar as imagens, no estádio fenológico de repouso, imagens 3 e 6 , observa-se as menores taxas de acerto, $50 \%$ e $45 \%$, respectivamente. Verifica-se que a inclusão dos atributos de textura melhorou o desempenho dos classificadores, uma vez que todas as classificações com textura resultaram em maior exatidão quando comparadas às classificações com atributos apenas espectrais. A estratificação da iluminação somada aos atributos texturais não incrementou a exatidão da classificação. Assim, infere-se que os atributos texturais e os dados de topografia estavam correlacionados, de modo que a inserção das classes de iluminação não forneceu ganho de informação à classificação das áreas cafeeiras, quando os atributos de textura já estavam presentes.

Nota-se também na Tabela 5 que a estratificação em cinco níveis de iluminação apresentou valores de exatidão muito próximos dos valores utilizando duas classes de iluminação, o que foi esperado uma vez que a inserção das classes de iluminação, não influenciou as exatidões das classificações, quando já havia atributos texturais. Ainda com base na Tabela 5, todos os estádios fenológicos, frutificação, granação e repouso apresentaram média de exatidão para a classe café de aproximadamente $69 \%$. Deste modo, não se pode afirmar que realizar o mapeamento em um determinado estádio fenológico da planta melhore o desempenho do mapeamento automático das áreas cafeeiras em imagens OLI/Landsat-8.

De modo geral, as classificações automáticas de áreas cafeeiras feitas para o município de Machado atingiram um máximo de $76 \%$ de exatidão para a classe café, valores mais altos que os encontrados na literatura para a mesma área de estudo utilizando a abordagem de classificação por redes neurais, que resultou em $63 \%$ de exatidão para a classe café (ANDRADE et al., 2012). Tal diferença ocorreu principalmente pela inserção dos atributos texturais. 
TABELA 5 - Índice global e exatidão da classe café das classificações baseadas em objetos em imagens OLI/ Landsat- 8 estratificada em cinco classes de iluminação, Machado (MG).

\begin{tabular}{|c|c|c|c|c|c|c|c|c|}
\hline \multirow{3}{*}{ Imagens } & \multicolumn{8}{|c|}{ Validação dos mapas temáticos de café do município de Machado (MG) } \\
\hline & \multicolumn{2}{|c|}{$\begin{array}{l}\text { Classificação com } \\
\text { atributos espectrais }\end{array}$} & \multicolumn{2}{|c|}{$\begin{array}{c}\text { Classificação com } \\
\text { atributos espectrais e } \\
\text { texturais }\end{array}$} & \multicolumn{2}{|c|}{$\begin{array}{l}\text { Classificação com } \\
\text { atributos espectrais, } \\
\text { texturais e } 2 \text { classes } \\
\text { de iluminação }\end{array}$} & \multicolumn{2}{|c|}{$\begin{array}{l}\text { Classificação com } \\
\text { atributos espectrais, } \\
\text { texturais e } 5 \text { classes } \\
\text { de iluminação }\end{array}$} \\
\hline & $\begin{array}{c}\text { Índice } \\
\text { global }(\%)\end{array}$ & $\begin{array}{l}\text { Exatidão } \\
\text { café }(\%)\end{array}$ & $\begin{array}{c}\text { Índice } \\
\text { global }(\%)\end{array}$ & $\begin{array}{l}\text { Exatidão } \\
\text { café }(\%)\end{array}$ & $\begin{array}{l}\text { Índice } \\
\text { global } \\
(\%)\end{array}$ & $\begin{array}{l}\text { Exatidão } \\
\text { café }(\%)\end{array}$ & $\begin{array}{c}\text { Índice } \\
\text { global } \\
(\%)\end{array}$ & $\begin{array}{l}\text { Exatidão } \\
\text { café }(\%)\end{array}$ \\
\hline $\begin{array}{c}\text { Imagem 1 } \\
(20 / 11 / 2013)\end{array}$ & 83 & 65 & 90 & 72 & 90 & 70 & 91 & 72 \\
\hline $\begin{array}{c}\text { Imagem } 2 \\
(08 / 02 / 2014)\end{array}$ & 86 & 59 & 91 & 74 & 90 & 73 & 91 & 76 \\
\hline $\begin{array}{c}\text { Imagem } 3 \\
(02 / 07 / 2014)\end{array}$ & 84 & 50 & 92 & 76 & 91 & 75 & 92 & 76 \\
\hline $\begin{array}{c}\text { Imagem } 4 \\
(07 / 11 / 2014)\end{array}$ & 86 & 58 & 91 & 74 & 90 & 71 & 90 & 75 \\
\hline $\begin{array}{c}\text { Imagem 5 } \\
(10 / 01 / 2015)\end{array}$ & 82 & 64 & 90 & 74 & 90 & 74 & 89 & 71 \\
\hline $\begin{array}{c}\text { Imagem } 6 \\
(21 / 07 / 2015)\end{array}$ & 80 & 45 & 90 & 73 & 90 & 72 & 90 & 70 \\
\hline Média & 83,5 & 56,8 & 90,7 & 73,8 & 90,2 & 72,5 & 90,5 & 73,3 \\
\hline
\end{tabular}

\section{CONCLUSÕES}

De acordo com a metodologia empregada, que utiliza dados de sensoriamento remoto e classificação automática baseada em objetos, associada a técnicas de mineração de dados, o café ocupa aproximadamente $22 \%$ do município de Machado, sul de Minas Gerais.

A utilização de atributos texturais no processo de classificação automática incrementa as taxas de exatidão de mapeamento na identificação de áreas cafeeiras em imagens OLI/Landsat-8.

Classificações automáticas, em imagens OLI/Landsat-8, que estratificam o relevo em duas ou cinco classes de iluminação, apresentam resultados semelhantes, tanto para exatidão da classe café quanto para índice global da imagem, desde que sejam utilizados atributos texturais.

Em imagens OLI/Landsat-8, por meio de classificação automática baseada em objetos, não houve diferença nos valores de exatidão da classe café que indicasse que um estádio fenológico (data) proporcionasse melhores resultados.

\section{AGRADECIMENTOS}

À Fundação de Coordenação de Aperfeiçoamento de Pessoal de Nível Superior (CAPES), pela bolsa concedida para o desenvolvimento desta pesquisa.

\section{REFERÊNCIAS}

ADAMI, M. et al. Fusão de imagens por IHS para melhorar a identificação de uso do solo em elementos amostrais. Engenharia Agrícola, Jaboticabal, v. 27, n. 2, p. 529-536, 2007.

ADDINK, E. A.; COILLIE, F. M. B. van; DE JONG, S. M. Introduction to the GEOBIA 2010 special issue: from pixels to geographic objects in remote sensing image analysis. International Journal of Applied Earth Observation and Geoinformation, Enschede, v. 15, p. 1-6, 2012.

ALI, A.; ALIREZA, S.; POURMANAFI, S. Crop type mapping in a highly fragmented and heterogeneous agricultural landscape: a case of central Iran using multi-temporal Landsat 8 imagery. Computers and Electronics in Agriculture, Athens, v. 127, p. 531540, 2016. 
ANDRADE, L. N. et al. Aplicação de redes neurais artificiais na classificação de áreas cafeeiras em Machado-MG. Coffee Science, Lavras, v. 8, n. 1, p. 78-90, 2012.

ANTUNES, A. F. B. Classificação de ambiente ciliar baseada em orientação a objeto em imagens de alta resolução espacial. 2003. 146 p. Tese (Doutorado em Ciências Geodésicas) - Universidade Federal do Paraná, Curitiba, 2003.

BAATZ, M.; SCHAPE, A. Multiresolution segmentation: an optimization approach for high quality multi-scale image segmentation. Angewandte Geographische Wichmann, v. 12, p. 12-23, 2000.

BANSKOTA, A. et al. Forest monitoring using Landsat time-series data: a review. Canadian Journal of Remote Sensing, Ottawa, v. 40, n. 5, p. 362-384, 2014.

BATTISTA, F.; FINO, D.; MANCINI, G. Optimization of biogas from coffee production waste. Bioresource Technology, Essex, v. 200, p. 884-890, 2016.

CALLIECO, F.; DELL'ACQUA, F. A comparison between two radiative transfer models for atmospheric correction over a wide range of wavelengths. International Journal of Remote Sensing, Abingdon, v. 32, n. 5, p. 1357-1370, 2011.

CAMARGO, A. Florescimento e frutificação de café arábica nas diferentes regiões (cafeeiras) do Brasil. Pesquisa Agropecuária Brasileira, Brasília, v. 20, n. 7, p. 831-839, jul. 1985.

CAMArgo, Â. P.; CAMArgo, M. B. P. Definição e esquematização das fases fenológicas do cafeeiro arábica nas condições tropicais do Brasil. Bragantia, Campinas, v. 60, n. 1, p. 65-68, 2001.

COMPANHIA NACIONAL DE ABASTECIMENTO. Levantamento café safra 2015. Brasília, DF, 2015. 43 p.

CORDERO-SANCHO, S.; SADER, S. A. Spectral analysis and classification accuracy of coffee crops using landsat and a topographic-environmental model. International Journal of Remote Sensing, Bristol, v. 28, n. 7, p. 1577-1593, Jan. 2007.

DELGADO, R. C. et al. Evolução espaço-temporal do café no estado de Minas Gerais por meio do classificador de árvore de decisão. Enciclopédia Biosfera, Goiânia, v. 8, n. 15, p. 904-913, 2012.
GAMANYA, R.; DE MAEYER, P.; DAPPER, M. An automated satellite image classification design using object-oriented segmentation algorithms: a move towards standardization. Expert Systems with Applications, Shreveport, v. 32, n. 2, p. 616-624, 2007.

GIROLAMO NETO, C. D. et al. Classificação automática de áreas cafeeiras utilizando imagens de sensoriamento remoto e técnicas de mineração de dados. In: SIMPÓSIO BRASILEIRO DE SENSORIAMENTO REMOTO, 2015, João Pessoa. Anais... São José dos Campos: INPE, 2015. p. 1609-1616.

GUHL, A. Café y cambio de paisaje en la zona cafetera colombiana entre 1970 y 1997. Cenicafe, Chinchiná, v. 55 , n. 1, p. 29-44, 2004.

HALL, M. et al. The WEKA data mining software. ACM SIGKDD Explorations Newsletter, New York, v. 11, n. 1, p. 10, 2009.

HARALICK, R.; SHANMUGAN, K.; DINSTEIN, I. Textural features for image classification. IEEE Transactions on Systems, Man, and Cybernetics, Macau, n. 6, p. 610-621, 1973.

INSTITUTO BRASILEIRO DE GEOGRAFIA E ESTATÍSTICA. Divisão regional do Brasil em mesorregiões e microrregiões geográficas. Rio de Janeiro, 1990. 135 p.

Produção agrícola municipal: culturas temporárias e permanentes 2012. Rio de Janeiro, 2012. Disponível em: <ftp://ftp.ibge. gov.br/Producao_Agricola/Producao_Agricola Municipal_\%5Banual\%5D/2012/pam2012.pdf $>$. Acesso: 11 ago. 2016.

INTERNATIONAL COFFEE ORGANIZATION. Total production by all exporting countries: 2015/2016. Disponível em: <http://www.ico.org/ historical/1990\%20onwards/PDF/1a-total-production. pdf>. Acesso em: 9 ago. 2016.

KIM, K. A hybrid algorithm by subspace partitioning through semi-supervised decision tree. Pattern Recognition, York, v. 60, p. 154-163, 2016.

LAMPARELLI, R. A. C.; NERY, L.; ROCHA, J. V. Utilização de técnicas por componentes principais (ACP) e fator de iluminação, no mapeamento da cultura do café em relevo montanhoso. Engenharia Agrícola, Jaboticabal, v. 31, n. 3, p. 584-597, 2011. 
LANDAU, D. P.; BINDER, K. A guide to MonteCarlo simulations in statistical physics. $3^{\text {rd }}$ ed. Cambridge: Cambridge University Press, 2009. 500 p.

LELONG, C. C. D.; CHANE, T. Application of textural analysis on very high resolution panchromatic images to map coffee orchards in Uganda. In: INTERNATIONAL GEOSCIENCE AND REMOTE SENSING SYMPOSIUM, 2003, Toulouse. Proceedings... Toulouse, 2003. v. 2, p. 1007-1009.

LI, Q. et al. Object-based crop classification with Landsat-MODIS enhanced time-series data. Remote Sensing, Basel, v. 7, p. 16091-16107, 2015.

LUDWIG, I. A. et al. Coffee: biochemistr and potential impact on health. Food \& Function, Cambridge, v. 5, p. 1695-1717, 2014.

LUZ, N. B. da; ANTUNES, A. F. B.; TAVARES JÚNIOR, J. B. Segmentação multirresolução e classificação orientada a objetos aplicados a imagens Spot-5 para o mapeamento do uso da terra. Floresta, Cabral, v. 40, n. 2, p. 429-446, 2010.

MACHADO, M. L. et al. Mapping of coffee lands (Coffea arabica L.) in the zona da mata region, Minas Gerais State, using remote sensing. Coffee Science, Lavras, v. 5, n. 2, p. 113-122, 2010.

MARUJO, R. F. B. Avaliação de métodos de segmentação de imagens aplicadas na classificação de culturas cafeeiras. 2013. 50 p. Monografia (Graduação em Ciência da Computação) - Universidade Federal de Lavras, Lavras, 2013.

MEYER, P. et al. Radiometric corrections of topographically induced effects on Landsat TM data in an alpine environment. ISPRS Journal of Photogrammetry and Remote Sensing, Abingdon, v. 48, n. 4, p. 17-28, 1993.

MOREIRA, E. P.; VALERIANO, M. M. Application and evaluation of topographic correction methods to improve land cover mapping using object-based classification. International Journal of Applied Earth Observation and Geoinformation, Enschede, v. 32, n. 1, p. 208-217, 2014.

MOREIRA, M. A.; BARROS, M. A.; RUDORFF, B. F. T. Geotecnologias no mapeamento da cultura do café em escala municipal. Sociedade e Natureza, Uberlândia, v. 20, n. 1, p. 101-110, 2008.
MOREIRA, M. A.; BERNARDES, T.; SAITO, N. S. Dados orbitais no contexto espaço-temporal da cafeicultura: estudo de caso: municípios de Alfenas e Machado, MG. In: SIMPÓSIO BRASILEIRO DE SENSORIAMENTO REMOTO, 16., 2013, Foz do Iguaçu. Anais... Foz do Iguaçu, 2013. p. 539-546.

MOREIRA, M. A. et al. Tecnologia de informação: imagens de satélite para o mapeamento de áreas de café de Minas Gerais. Informe Agropecuário, Belo Horizonte, v. 28, n. 241, p. 27-37, 2007.

MOURA, L. C. et al. Levantamento e mapeamento espaço: temporal dos cafezais no município mineiro de Machado. Caminhos de Geografia, Uberlândia, v. 33, p. 3-8, 2009.

PEÑA, M. A.; BRENNING, A. Assessing fruit-tree crop classification from Landsat-8 time series for the Maipo Valle, Chile. Remote Sensing of Environment, New York, v v. 171, p. 234-244, 2015.

PESCK, V. A.; DISPERATI, A. A.; SANTOS, J. R. dos. Comparação das técnicas de fusão aplicadas à imagem Quickbird-2. Floresta e Meio Ambiente, Seropédica, v. 18, n. 2, p. 127-134, 2011.

PRATT, W. Digital image processing: PIKS scientific inside. $4^{\text {th }}$ ed. New York: J. Wiley, 2007. 786 p.

QUINLAN, J. C4. 5: programs for machine learning. San Mateo: M. Kaufmann, 1993. 302 p.

ROSA, V. G. C. Modelo agrometeorológico-espectral para monitoramento e estimativa da produtividade do café na Região Sul / Sudoeste do Estado de Minas Gerais. 2007. 142 p. Dissertação (Mestrado em Sensoriamento Remoto) - Instituto Nacional de Pesquisas Espaciais, São José dos Campos, 2007.

SANTOS, J. A. dos et al. Multiscale classification of remote sensing images. IEEE Transactions on Geoscience and Remote Sensing, Caceres, v. 50, n. 10, p. 3764-3775, 2012.

SARMIENTO, C. M. et al. Comparação de classificadores supervisionados na discriminação de áreas cafeeiras em Campos Gerais, Minas Gerais. Coffee Science, Lavras, v. 9, n. 4, p. 546-557, 2014.

SCHULTZ, B. et al. Data mining and object based image analysis applied to soybean areas classification through timeseries TM/ETM+ images. In: GEOGRAPHIC OBJECT-BASED IMAGE ANALYSIS CONFERENCE, 5., 2014, Thessaloniki. Proceedings... Thessaloniki, 2014. v. 4, p. 122-122. 
SILVA JUNIOR, C.; FRANK, T.; RODRIGUES, T. Discriminação de áreas de soja por meio de imagens EVI/MODIS e análise baseada em geo-objeto. Revista Brasileira de Engenharia Agrícola e Ambiental, Campina Grande, v. 18, n. 1, p. 44-53, 2014.

SONG, C. et al. Classification and change detection using Landsat TM data: when and how to correct atmospheric effects? Remote Sensing of Environment, New York, v. 75, p. 230-244, 2001.

SOUZA, V. C. O. et al. Espacialização e dinâmica da cafeicultura mineira entre 1990 e 2008, utilizando técnicas de geoprocessamento. Coffee Science, Lavras, v. 7, n. 2, p. 122-134, 2012.

VALERIANO, M. D. M. Cálculo do fator topográfico de iluminação solar para modelagem ecofisiológica a partir do processamento de Modelos Digitais de Elevação (MDE). In: SIMPÓSIO BRASILEIRO DE SENSORIAMENTO REMOTO, 15., 2011, Curitiba. Anais... Curitiba, 2011. p. 5933-5940.
VERDUZCO, G. C.; PIZAÑA, J. M.; BELLO, G. M. C. Coupling community mapping and supervised classification to discriminate Shade coffee from Natural vegetation. Applied Geography, Abingdon, v. 34, p. $1-9,2012$.

VIEIRA, M. A. et al. Series to map sugarcane over large areas. Remote Sensing of Environment, Lavras, v. 123, p. 553-562, 2012.

VIEIRA, T. G. C. et al. Geothecnologies in the assessment of land use changes in coffee regions of the state of Minas Gerais in Brazil. Coffee Science, Lavras, v. 2, n. 2, p. 142-149, 2006.

XIE, Y.; SHA, Z.; YU, M. Remote sensing imagery in vegetation mapping: a review. Journal of Plant Ecology, Oxford, v. 1, n. 1, p. 9-23, 2008.

ZHOU, Z. et al. Object-oriented classification of sugarcane using time-series middle-resolution remote sensing data based on AdaBoost. PLoS One, San Francisco, v. 10, n. 11, p. 1-16, 2015. 